\title{
Review of the weak mixing angle results with heavy quarks at LEP and SLC
}

\author{
Britta Schwering (Überschär) \\ BUGH-Wuppertal; Gaußstr. 20, D-42097 Wuppertal \\ E-mail: 'Britta.Schwering@cern.ch'
}

ABSTRACT: Recent precise measurements of the weak mixing angle, $\sin ^{2} \theta_{\text {eff }}^{\text {lep }}$, by the SLD experiment at SLC and by the ALEPH, DELPHI, L3 and OPAL experiments at LEP are reviewed. Emphasis is put on the determination of $\sin ^{2} \theta_{\mathrm{eff}}^{\text {lep }}$ with measurements of the heavy quark asymmetry at the $\mathrm{Z}$ pole. The techniques of flavour tagging are discussed and the different methods of asymmetry measurements are presented. As an example the jet charge technique is explained in more detail.

\section{Introduction}

Experiments at LEP and SLC are providing increasingly precise measurements of electroweak parameters, eg. the couplings of the $\mathrm{Z}$ to fermions and $\sin ^{2} \theta_{\text {eff }}^{\text {lep }}$.

LEP is an $\mathrm{e}^{+} \mathrm{e}^{-}$-collider with four experiments, ALEPH, DELPHI, L3 and OPAL. During the LEP I phase from 1989 until 1995 the centre-of-mass energy was adjusted to be close to the mass of the $\mathrm{Z}$ boson. About four million $\mathrm{Z}$ decays have been observed by each experiment. The measurement of the beam energy reached a precision of a few MeV. Since 1995 the beam energy has been increased in order to measure $\mathrm{W}$ pair production or triple gauge couplings

SLC is an $\mathrm{e}^{+} \mathrm{e}^{-}$-linear collider with one experiment SLD. It is running as well close to the $\mathrm{Z}$ pole and has delivered more than 300000 events. SLC has the advantage of a small beampipe and a small beam size. The lack in statistics is compensated by the possibility to polarize the electron beam up to $80 \%$.

\section{Observables at the $\mathrm{Z}$}

In $\mathrm{e}^{+} \mathrm{e}^{-}$-annihilation four observables can be measured for any given fermion: the total cross section, the forward-backward asymmetry and the final state polarization together with its forward- backward asymmetry $[\overline{2} \bar{\eta}$. In the case of final state quarks, the two latter are not accessible. Strong decays conserve parity, while weak decays destroy the polarization information because they occur in general from scalar meson states. If the initial state beam can be polarized additional information can be measured according to the different beam polarization.

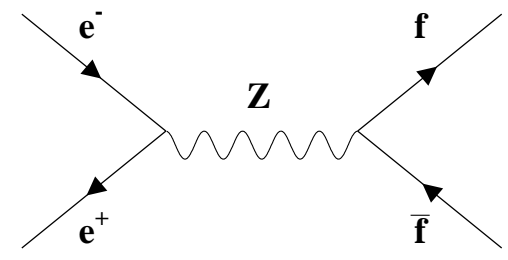

Figure 1: $\mathrm{e}^{+} \mathrm{e}^{-}-$annihilation, only the dominant LEP/SLC Born level diagram is pictured.

The most precise results of $\sin ^{2} \theta_{\text {eff }}^{\text {lep }}$ are deduced from asymmetry measurements with heavy quarks which are performed at the $\mathrm{Z}$ pole. Close to the $\mathrm{Z}$ resonance the $\mathrm{Z}$ exchange contributes 100 times more than the photon exchange. Exactly on the $\mathrm{Z}$ peak , the $\mathrm{Z}$ exchange amplitude is imaginary and the $\gamma-Z$ interference vanishes. As a result only small corrections coming from the interference term and the photon exchange are expected and have to be applied. The dominant Feynmann diagram is shown in Fig. 11. 


\section{Asymmetries}

\subsection{Definitions of the observables at Born level}

The differential cross section $\mathrm{e}^{+} \mathrm{e}^{-} \rightarrow \mathrm{f} \overline{\mathrm{f}}(\mathrm{f} \neq \mathrm{e})$ in Born approximation for massless fermions and unpolarized beams is given by:

$$
\begin{aligned}
\frac{d \sigma_{\mathrm{f}}(s)}{d \cos \theta}= & \frac{N_{C}^{\mathrm{f}} G_{F}^{2} m_{\mathrm{Z}}^{4}}{16 \pi} \frac{s}{\left(s-m_{\mathrm{Z}}^{2}\right)^{2}+\frac{s^{2}}{m_{\mathrm{Z}}^{2}} \Gamma_{\mathrm{Z}}^{2}} \times \\
& {\left[\left(v_{\mathrm{e}}^{2}+a_{\mathrm{e}}^{2}\right)\left(v_{\mathrm{f}}^{2}+a_{\mathrm{f}}^{2}\right)\left(1+\cos ^{2} \theta\right)+\right.} \\
& \left.2 v_{\mathrm{e}} a_{\mathrm{e}} v_{\mathrm{f}} a_{\mathrm{f}} \cos \theta\right]
\end{aligned}
$$

Note that final state electrons are not considered, because of their t-channel contribution. $\sqrt{s}$ is the centre-of-mass energy, $m_{\mathrm{Z}}$ is the mass of the $\mathrm{Z}$, $\Gamma_{\mathrm{Z}}$ is the width of the $\mathrm{Z}$ and $G_{F}$ is the Fermi coupling constant. The colour factor, $N_{C}^{\mathrm{f}}$, is 1 for leptons and 3 for quarks. $\cos \theta$ is the angle between the incoming electron and the outgoing fermion.

Important is the appearance of the axial vector and the vector coupling of the $\mathrm{Z}$ to the fermion:

$$
\begin{aligned}
& a_{\mathrm{f}}=i_{3}^{\mathrm{f}} \\
& v_{\mathrm{f}}=a_{\mathrm{f}}\left(1-4\left|q_{\mathrm{f}}\right| \sin ^{2} \theta_{W}\right)
\end{aligned}
$$

Where $i_{3}^{\mathrm{f}}$ is the third component of the weak isospin, $q_{\mathrm{f}}$ the fermion charge and $\sin ^{2} \theta_{W}$ the weak mixing angle.

The forward-backward asymmetry is defined via the differential forward, $\sigma_{F}^{\mathrm{f}}$, and backward, $\sigma_{B}^{\mathrm{f}}$, cross section

$$
A_{F B}^{\mathrm{f}}=\frac{\sigma_{F}^{\mathrm{f}}-\sigma_{B}^{\mathrm{f}}}{\sigma_{F}^{\mathrm{f}}+\sigma_{B}^{\mathrm{f}}}
$$

and can be expressed in terms of the axial vector and vector couplings:

$$
A_{F B}^{\mathrm{f}}=\frac{3}{4} \mathcal{A}_{\mathrm{e}} \mathcal{A}_{\mathrm{f}}, \quad \text { with } \mathcal{A}_{\mathrm{f}}=\frac{2 v_{\mathrm{f}} a_{\mathrm{f}}}{v_{\mathrm{f}}^{2}+a_{\mathrm{f}}^{2}} .
$$

These $\mathcal{A}_{\mathrm{f}}$ factors are shown in Fig ${ }_{2} \overline{2}$ as a function of $\sin ^{2} \theta_{W}$ for leptons, up type and down type quarks.

$\mathcal{A}_{\mathrm{f}}$ for leptons varies strongly with $\sin ^{2} \theta_{W}$, while it is nearly one and stable for down type quarks and shows little dependence for up type

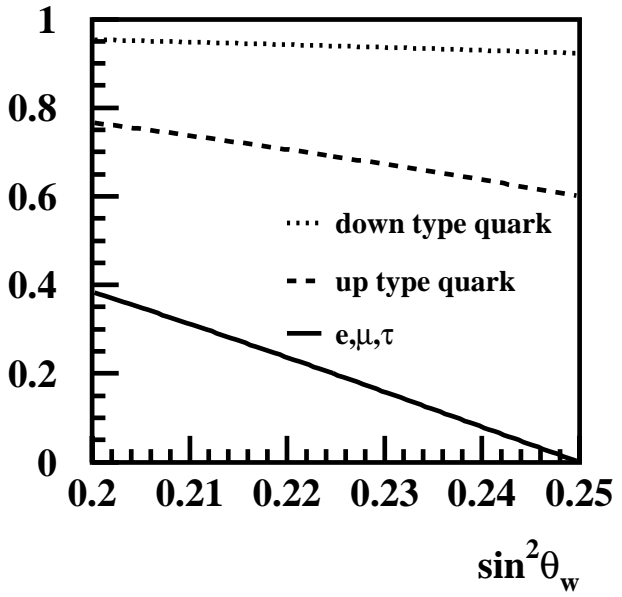

Figure 2: $\mathcal{A}_{\mathrm{f}}$ as a function of $\sin ^{2} \theta_{W}$ for leptons, up type and down type quarks.

quarks. As a result the forward-backward asymmetry measured for b and c quarks is mostly sensitive to the initial state couplings of the electron to the $\mathrm{Z}$.

In case of polarized electron beams, with polarization $\mathcal{P}$, a left-right asymmetry can be defined as:

$$
A_{L R}=\frac{1}{\mathcal{P}} \frac{\sigma_{L}-\sigma_{R}}{\sigma_{L}+\sigma_{R}}
$$

which is in terms of the couplings only sensitive to the electron couplings to the $\mathrm{Z}$ and independent on the final state couplings

$$
A_{L R}=\mathcal{A}_{\mathrm{e}} .
$$

The forward-backward asymmetry for polarized beams

$$
A_{F B}^{\mathrm{f}}=\frac{3}{4} \mathcal{A}_{\mathrm{f}} \frac{\mathcal{A}_{\mathrm{e}}-\mathcal{P}}{1-\mathcal{A}_{\mathrm{e}} \mathcal{P}}
$$

is as well sensitive to the initial state couplings but modified by the amount of polarization.

Finally the left-right-forward-backward asymmetry can be constructed:

$$
\begin{aligned}
A_{L R, F B}^{\mathrm{f}} & =\frac{1}{\mathcal{P}} \frac{\left[\sigma_{L, F}^{\mathrm{f}}-\sigma_{L, B}^{\mathrm{f}}\right]-\left[\sigma_{R, F}^{\mathrm{f}}-\sigma_{R, B}^{\mathrm{f}}\right]}{\sigma_{L}^{\mathrm{f}}+\sigma_{R}^{\mathrm{f}}} \\
& =\frac{3}{4} \mathcal{A}_{\mathrm{f}}
\end{aligned}
$$

which is only sensitive to the final state couplings. 


\subsection{Radiative Corrections}

Apart from corrections for the $\gamma-\mathrm{Z}$ interference and the photon exchange, additional corrections have to be applied to the born level formulas. They can be divided into three classes [i2]]:

- photonic corrections : These are corrections coming from processes where an additional photon is attached at any place to the Born level diagram. The largest amount is coming from initial state radiation, which can be reliably calculated in QED. Close to the $\mathrm{Z}$ resonance most of the radiations are soft and therefore the kinematics of the events are only slightly distorted.

- QCD corrections : They originate in first order from gluon radiation. For the total cross sections they are calculated to third order $\alpha_{s}$. The kinematics of an event is distorted seriously in case of hard gluon radiation and the influences can be calculated using second order QCD matrix elements or parton shower Monte Carlo programs. Note that the contribution to the different analyses varies and strongly depends on the method used.

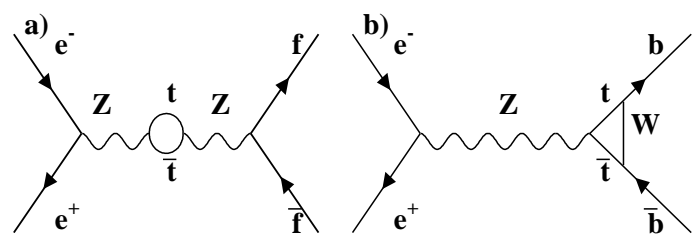

Figure 3: Example of electroweak corrections a) correction to the $\mathrm{Z}$ propagator, $\mathrm{b}$ ) correction to the $\mathrm{Zb} \overline{\mathrm{b}}$ vertex.

- genuine electroweak corrections : These are corrections coming from loop diagrams (see Fig 3 a) ) or corrections to the vertex (see Fig b) ). They can be parameterized in very good approximation by effective couplings

$$
\begin{aligned}
& \bar{a}_{\mathrm{f}}=\sqrt{1+\Delta \rho_{\mathrm{f}}} a_{\mathrm{f}} \\
& \bar{v}_{\mathrm{f}}=\bar{a}_{\mathrm{f}}\left(1-4\left|q_{\mathrm{f}}\right| \sin ^{2} \theta_{\mathrm{eff}}^{\mathrm{f}}\right),
\end{aligned}
$$

which than substitute $a_{\mathrm{f}}$ and $v_{\mathrm{f}}$ in the Born level formulas. Theoretically they are of interest, because new particles would contribute to these corrections. If one assumes lepton universality all leptonic observables which can be measured at LEP and SLC can be described by the effective mixing angle $\sin ^{2} \theta_{\text {eff }}^{\text {lep }}$ and $\Delta \rho_{\text {lep }}$.

\subsection{The b quark sector}

As mentioned before the forward-backward asymmetry for b quarks, as a down type quark, is most sensitive to the $\sin ^{2} \theta_{W}$. The sensitivity at Born level is depicted in Fig.tili for leptons, up type and down type quarks separately.

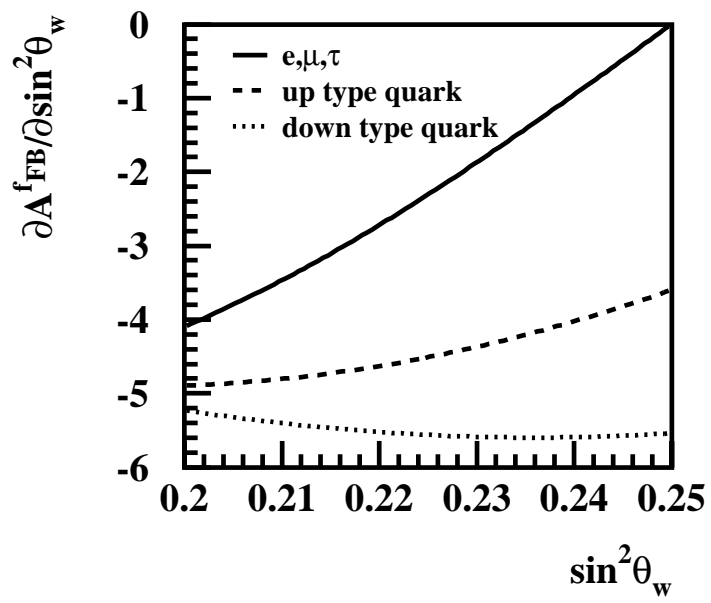

Figure 4: Sensitivity of the forward-backward asymmetry for leptons, up type and down type quarks.

When applying the necessary corrections and switching to the effective couplings the measurement of $b$ asymmetries still gives the most precise measurement of $\sin ^{2} \theta_{\text {eff }}^{\text {lep }}$.

The $b$ quark is the isospin partner of the heavy top quark, which leads to additional electroweak corrections, i.e. vertex corrections (Fig. $\overline{3}$ ! b) ). Therefore in first place Higgs or hypothetical Higgs-like particles, which couple to the mass, will be seen in the $\mathrm{b}$ quark sector.

As a consequence the measurement of $\mathrm{b}$ asymmetries is sensitive to $\log \left(m_{\text {Higgs }}\right)$, to new particles, and provides one of the most precise measurement of $\sin ^{2} \theta_{\text {eff }}^{\text {lep }}$. 


\section{Flavour Tagging}

The tagging of heavy quarks, namely $\mathrm{c}$ and $\mathrm{b}$ quarks, is possible because of three different features:

- $\mathrm{b}$ and $\mathrm{c}$ quarks decay weakly. Therefore $\mathrm{C}$ and B Hadrons have lifetime and their decay vertex can be separated from the primary vertex. The need of precise vertex detectors is obvious.

- $\mathrm{b}$ and $\mathrm{c}$ quarks decay semileptonic. Per lepton species the branching ratio is about $10 \%$. Good muon and electron identification is necessary to use this statistically limited data sample.

- $\mathrm{C}$ hadrons can be reconstructed via their decay products. Because of the cascade decay, $\mathrm{b} \rightarrow \mathrm{c} \rightarrow \mathrm{X}$, the reconstruction of $\mathrm{D}$ mesons enriches also the $\mathrm{b}$ quark content. For the D meson reconstruction, particle identification is crucial.

It is important to distinguish between the two quark flavours to measure an asymmetry for $\mathrm{b}$ and $\mathrm{c}$ quarks separately. In the tagging based on lifetime or semileptonic decays this is performed by using for example the measurement of the mass difference at the secondary vertex. In addition, other features of the decay products as the transverse momentum or the charged multiplicity at the secondary vertex can be used.

In contrary, when the tagging is performed by reconstruction of $\mathrm{D}$ mesons, the $\mathrm{C}$ hadron energy or the lifetime information is used to separate $\mathrm{c}$ from $\mathrm{b}$ quarks.

\subsection{Different flavour tagging methods}

- The classical method is the lepton tag. It is used by all four LEP experiments to measure the forward-backward asymmetry. In semileptonic decays the transverse momentum of the lepton can reach maximal half of the hadrons mass. As the direction of the hadron can be approximated by the jet direction, the measurement of the lepton's transverse momentum with respect to this jet axis can be used as tagging variable. A high $p_{t}$ indicates a heavy final state quark.
- The lifetime information is used mainly in three different ways for heavy quark tagging.

The first approach is based on the signed impact parameter measurement. It was invented by ALEPH and adopted by DELPHI and L3. Starting from the impact parameter measurement for each track in an event a significance is calculated and transformed into a probability. This probability is then combined to either an event tag, a hemisphere tag or a jet tag, depending on the method used. The later is used for gluon splitting analyses. The hemisphere tag allows to measure the efficiency and the purity of b quarks with the double tag method and is e.g. applied in measurements of $R_{\mathrm{b}}$. The event tag reaches the best efficiency at a given purity and is used for asymmetry measurements.

In a second approach the secondary vertex itself is reconstructed. This procedure is used by OPAL and SLD. The distance between the primary and the secondary vertex divided by its corresponding error is used as tagging variable. The lifetime inside $b$ events leads to well sepa-

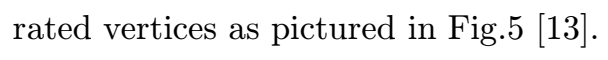

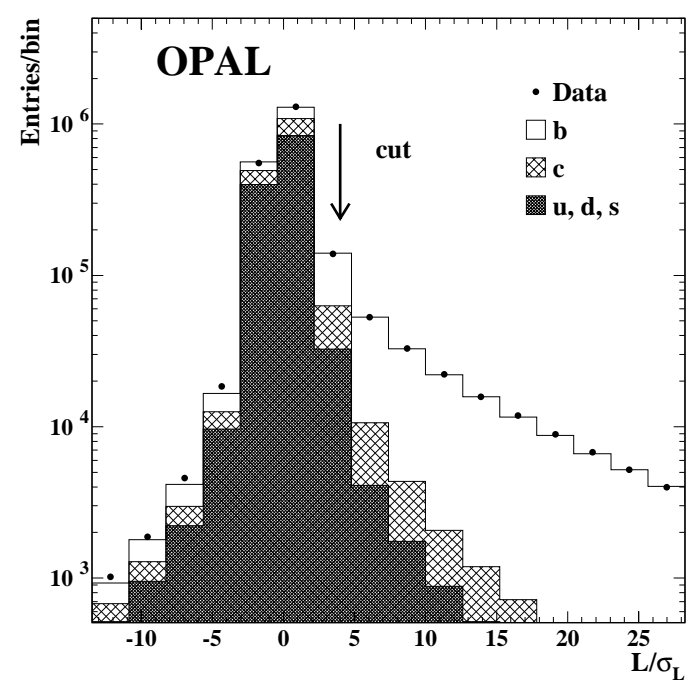

Figure 5: The $\mathrm{b}$ tagging variable, $\mathrm{L} / \sigma_{\mathrm{L}}$, in comparison of data and simulation measured by OPAL.

The third approach was developed from the impact parameter method. The big enhancement is coming from the measurement, of the invariant mass of the particles at the secondary vertex, included. 


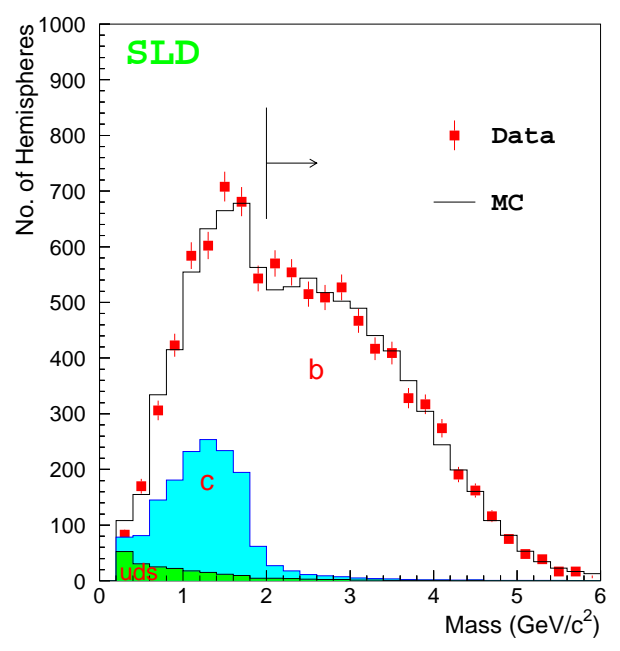

Figure 6: The corrected mass distribution in comparison of data and simulation measured by SLD.

This method is used by ALEPH, DELPHI and SLD. The mass distribution in comparison of data and simulation for SLD is shown in Fig. $\overline{6}_{1}$ [199], where a good separation between $\mathrm{c}$ and $\mathrm{b}$ quarks can be seen.

The transverse momentum measurement is included in the procedure used by SLD, while DELPHI uses the rapidity of secondary particles and the energy of the decay particles. The gain in the b efficiency versus b purity plane is shown

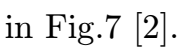

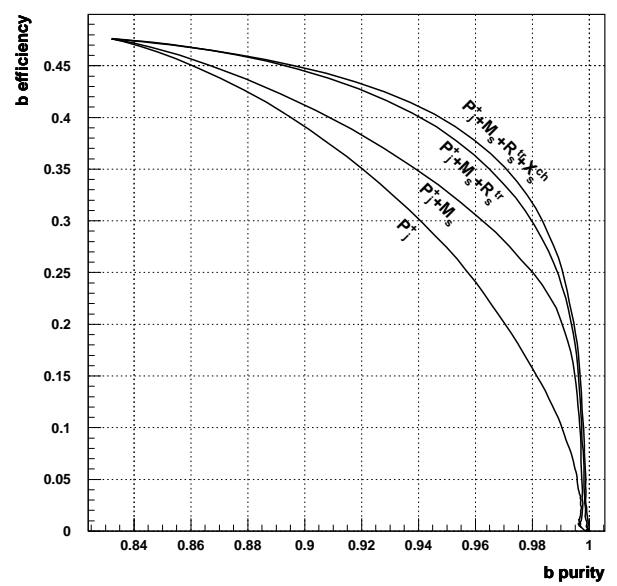

Figure 7: b efficiency as a function of b purity as measured by DELPHI. The gain including the different observables is shown.
As a summary the performance of the hemi-

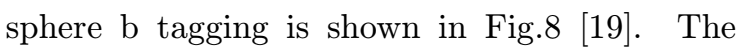
working points for the variuos tagging procedures used are given for the different experiments.

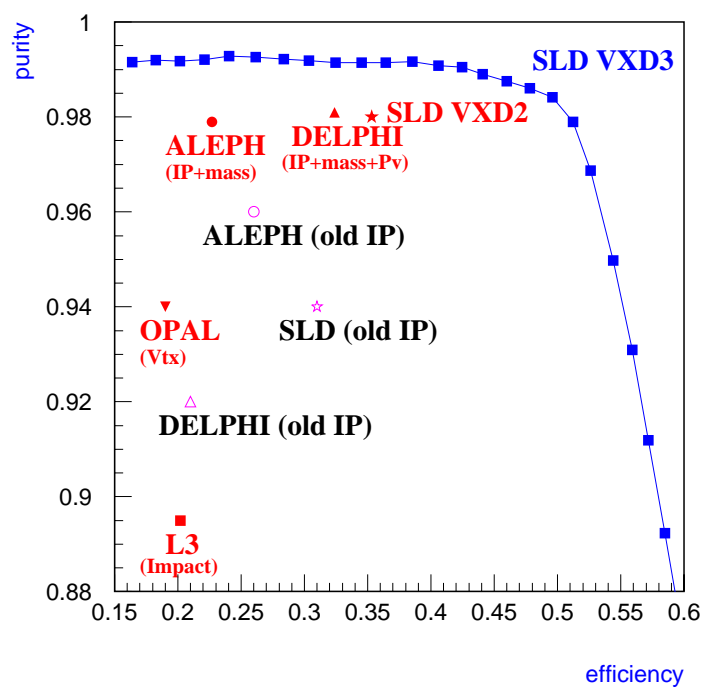

Figure 8: The performance of the hemisphere b tagging for different experiments.

- The last tagging method is based on the reconstruction of $\mathrm{D}$ mesons. They can be reconstructed

- exclusive by: $\mathrm{D}^{0}, \mathrm{D}^{+}, \mathrm{D}_{\mathrm{s}}$ and $\Lambda_{\mathrm{c}}$

- semi-exclusive by: $\mathrm{D}^{*+}$

- inclusive by: $\mathrm{D}^{*+}$.

This procedure enriches primary c and b quark events, because heavy quark production is suppressed in the fragmentation.

The separation between $\mathrm{c}$ and $\mathrm{b}$ quark events is performed using either the lifetime information, as described above, or measurements as the energy of the $\mathrm{C}$ hadron or its corresponding decay length.

\section{LEP - Asymmetry Measurements}

Good performance of the flavour tagging is crucial for an asymmetry measurement, since the control of the background, with sometimes different corresponding asymmetry behaviour, is needed. It is also important to distinguish between the 
quark and the anti-quark hemisphere. The measurement of the forward-backward asymmetry can be diluted by $\overline{\mathrm{B}^{0}}-\mathrm{B}^{0}$ mixing, charge mistag or wrong estimation of the background.

\subsection{Lepton tag method}
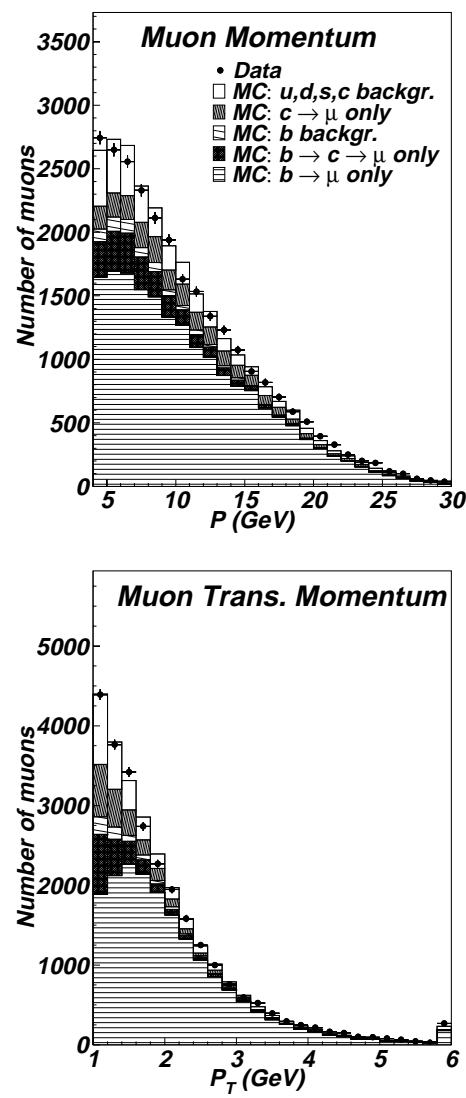

Figure 9: The momentum and transverse momentum spectra for muons measured by L3 in comparison of data and simulation.

In the classiysis the lepton is used as tag for the heavy quarks and to distinguish between quark and anti-quark. In the direct decay $b \rightarrow 1$ the lepton has negative charge, while in the c case, the lepton has positive charge. Therefore the cascade decay $\mathrm{b} \rightarrow \mathrm{c} \rightarrow \mathrm{l}$ has to be treated separately. ses are shown in

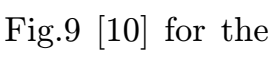
of muons as measured by L3. As mentum, $p$, and tum, $p_{t}$, distributions are shown.

In the case of cal lepton tag analThe contribution of different decay classemileptonic decay an example the motransverse momen$\overline{\mathrm{B}}^{0}-\mathrm{B}^{0}$ mixing the charge correlation is mirrored and an additional correction has to be applied.

Systematic uncertainties are introduced by the mixing, the semileptonic branching ratios and the modelling of the lepton spectra. Some of the uncertainties can be reduced, when fitting the forward-backward asymmetry for $\mathrm{c}$ and b quarks together with the mixing parameter $\bar{\chi}$. ALEPH and L3 are using only high $p_{t}$ leptons and deduce the forward-backward asymmetry for b quarks only. DELPHI, OPAL and SLD are using the full $\left(p, p_{t}\right)$ spectrum and obtain a result for both

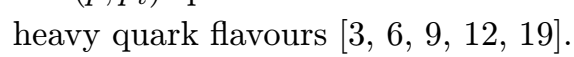

\subsection{D meson reconstruction method}

The measurement of the forward-backward asymmetries for heavy quarks using reconstructed D mesons is from the strategy similar to the lepton tag method. The reconstructed D meson is used for tagging the heavy quark and for identifying the quark and the anti-quark. For the asymmetry measurement only $\mathrm{D}^{*+}, \mathrm{D}^{0}$ and $\mathrm{D}^{+}$ are reconstructed, but up to 9 channels can be used and enlarge the statistics (see for example Fig. 10

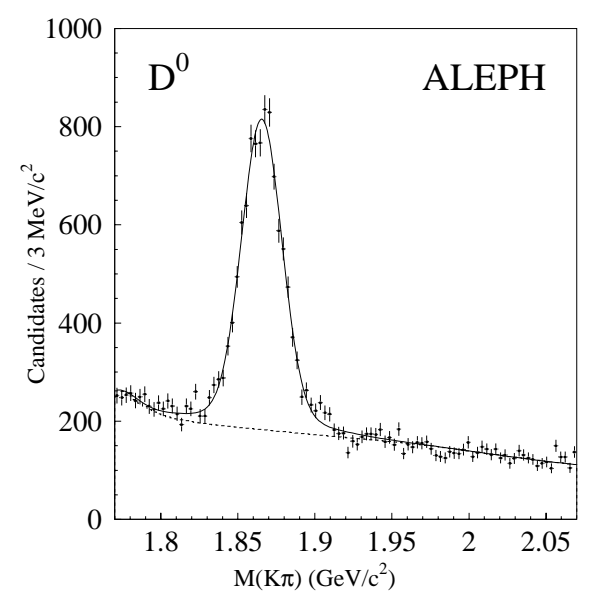

Figure 10: The invariant mass distribution for one $\mathrm{D}^{0}$ decay channel as measured by ALEPH.

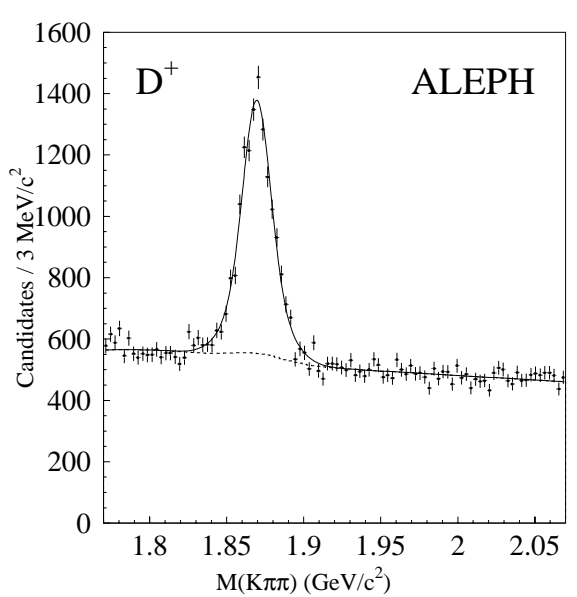

Figure 11: The invariant mass distribution for one $\mathrm{D}^{+}$decay channel as measured by ALEPH.

The charge measurement of the $\mathrm{D}$ decay products are correlated with the initial state quark 
and are used to identify the quark or the antiquark hemisphere. Like in the lepton case, the $\overline{\mathrm{B}}^{0}-\mathrm{B}^{0}$ mixing will dilute the charge correlation. It has been measured, that the production of $\mathrm{D}$ mesons in the upper vertex, which is coming from the $\mathrm{W}$ decay, is not negligible and a correction compatible to the mixing has to be applied. When combining the lepton and the D information the error can be reduced by their opposite charge correlation. Clearly this method is more sensitive by a factor two to the $\mathrm{c}$ forwardbackward asymmetry than to the b. A hard cut into the momentum of the reconstructed D meson reduces the $\mathrm{b}$ event contribution as applied by ALEPH and a measurement of the c forwardbackward asymmetry is performed. DELPHI and OPAL apply a softer selection and both the $\mathrm{c}$ and b forward-backward asymmetries are deduced isis.

\subsection{The jet charge method}

This approach uses the hemisphere charge

$$
Q_{F(B)}=\frac{\sum_{i} q_{i}\left|\overrightarrow{p_{i}} \cdot \vec{T}\right|^{\kappa}}{\sum_{i}\left|\overrightarrow{p_{i}} \cdot \vec{T}\right|^{\kappa}} \quad \begin{aligned}
& F: \overrightarrow{p_{i}} \cdot \vec{T}>0 \\
& B: \overrightarrow{p_{i}} \cdot \vec{T}<0
\end{aligned}
$$

to distinguish between the quark and the antiquark. The event is divided by the Thrust axis, $\vec{T}$, into two hemispheres and all particles are assigned with respect to this axis either as forward or backward. To build $Q_{F(B)}$, the charge of one particle, $q_{i}$ is weighted by its longitudinal momentum, $\left|\overrightarrow{p_{i}} \cdot \vec{T}\right|$, decorated with an exponent $\kappa$, which is used to optimize the correlation between the hemisphere charge and the underlying quark charge.

For every event two observables charge flow, $Q_{F B}$, and total charge, $Q_{T O T}$, can be defined:

$$
Q_{F B}=Q_{F}-Q_{B} \quad ; \quad Q_{T O T}=Q_{F}+Q_{B} .
$$

A single measurement of the hemisphere charge of the quark and the anti-quark can be regarded as sum of three independent terms [i]i]:

$$
Q_{\mathrm{f}}=\frac{\delta_{\mathrm{f}}}{2}+\frac{E_{\mathrm{f}}}{2}+S_{\mathrm{f}} ; Q_{\overline{\mathrm{f}}}=\frac{-\delta_{\mathrm{f}}}{2}+\frac{E_{\overline{\mathrm{f}}}}{2}+S_{\overline{\mathrm{f}}},(5.1
$$

where $E_{\mathrm{f}}\left(=E_{\overline{\mathrm{f}}}\right)$ is a non vanishing (positive) bias due to hadronic re-interactions in the detector material. $S_{\mathrm{f}(\overline{\mathrm{f}})}$ accounts for statistical variations of $Q_{\mathrm{f}(\overline{\mathrm{f}})}$, thus $\left\langle S_{\mathrm{f}(\overline{\mathrm{f}})}\right\rangle=0$.
The charge separation $\delta_{\mathrm{f}}$ is defined by the charge flow of the hemisphere charge of the quark and the anti-quark:

$$
\delta_{\mathrm{f}}=\left\langle Q_{\mathrm{f}}-Q_{\overline{\mathrm{f}}}\right\rangle .
$$

Except for detector influences (mainly hadronic re-interactions), the average total charge, $\left\langle Q_{T O T}\right\rangle$, is expected to vanish. For a pure sample of one flavour $f$, the charge flow is related to the quark asymmetry via the charge separation:

$$
\left\langle Q_{F B}^{\mathrm{f} \overline{\mathrm{f}}}\right\rangle=\delta_{\mathrm{f}} A_{F B}^{\mathrm{f} \overline{\mathrm{f}}},
$$

while the relation is more complicated for a flavour mixture

$$
\left\langle Q_{F B}\right\rangle=\sum_{\mathrm{f}} \eta_{\mathrm{f}} \delta_{\mathrm{f}} P_{\mathrm{f}} A_{F B}^{\mathrm{f} \bar{f}} .
$$

The relative abundance of a quark flavour $f$ in the hadronic event sample is $P_{\mathrm{f}}$. The weighting factor $\eta_{\mathrm{f}}$ accounts mainly for the incomplete angular acceptance, limited angular resolution and other detector effects, but also for effects of gluon radiation.

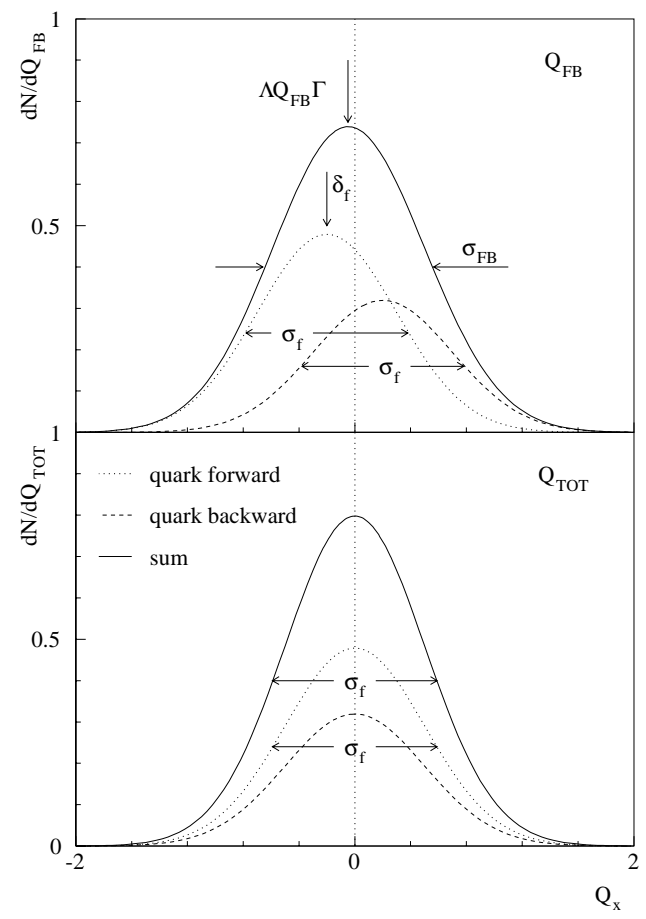

Figure 12: Sketch of the principle of the $\left\langle Q_{F B}\right\rangle$ and the $\delta_{\mathrm{f}}$ measurement for a single (down type) flavour f. 
In Fig. $122_{-1}^{\prime}$ the principle of the $\left\langle Q_{F B}\right\rangle$ and the $\delta_{\mathrm{f}}$ measurement for a single (down type) flavour $\mathrm{f}$ is shown. Note that the RMS of all distributions except the charge flow are equal. $\delta_{\mathrm{f}}$ is strongly related to the difference of the variances of the charge flow and the total charge distributions $[\overline{7} \overline{1} \mid$.

Using the event $b$ tag, which is based on the enhanced impact parameter method, the $\mathrm{b}$ efficiency and the b purity can be measured mainly from data, e.g. with the following formulas (DELPHI):

$$
\epsilon_{\mathrm{b}}(c)=\frac{\mathcal{F}(c)-R_{\mathrm{c}} \times \epsilon_{\mathrm{c}}(c)-R_{\mathrm{dus}} \times \epsilon_{\mathrm{dus}}(c)}{R_{\mathrm{b}}},
$$

where $\mathcal{F}(c)$ is the fraction of selected events at a given $\mathrm{b}$ tag cut value and measured from the data. $\epsilon_{\text {dus }}$ and $\epsilon_{\mathrm{c}}$ are the selection efficiencies for the light flavours and the charm events, which are both obtained from the simulation. The fractions of $c \bar{c}$ and $b \bar{b}$ events produced in hadronic $\mathrm{Z}^{0}$ decays, $R_{\mathrm{c}}$ and $R_{\mathrm{b}}$, are fixed to their Standard Model values. Thus $R_{\text {dus }}$ is equal to $\left(1-R_{\mathrm{c}}-R_{\mathrm{b}}\right)$. The corresponding purities can be calculated as

$$
P_{\mathrm{f}}(c)=\epsilon_{\mathrm{f}}(c) \times \frac{R_{\mathrm{f}}}{\mathcal{F}(c)} .
$$

Similar procedures are used by the other experiments to extract either the $\mathrm{b}$ or the light mistag from data.

The extraction of the $\mathrm{b}$ charge separation from the data is one of the important items of the jet charge method. This avoids a dependence on the poorly known B hadron decays. Only a small correction coming mainly from the hemispherehemisphere correlation has to be taken from simulation. The mixing and most of the QCD corrections are included in the measurement.

From the hemisphere charge for the quark and the anti-quark (Eqn.5.1.) a formula can be deduced, which relates the $\mathrm{b}$ charge separation and other measurable quantities:

$$
\begin{aligned}
\sum_{\mathrm{f}=\mathrm{d}, \mathrm{u}, \mathrm{s}, \mathrm{c}, \mathrm{b}} P_{\mathrm{f}} \delta_{\mathrm{f}}^{2} & =\sigma_{F B}^{2}-\sigma_{T O T}^{2}+\left\langle Q_{F B}\right\rangle^{2} \\
& \underbrace{+\sum_{\mathrm{f}=\mathrm{d}, \mathrm{u}, \mathrm{s}, \mathrm{c}, \mathrm{b}} P_{\mathrm{f}}\left(4\left\langle S_{\mathrm{f}} S_{\overline{\mathrm{f}}}\right\rangle+E_{\mathrm{f}}^{2}\right)-\left(\sum_{\mathrm{f}, \mathrm{s}, \mathrm{c}, \mathrm{b}} P_{\mathrm{f}} E_{\mathrm{f}}\right)^{2}}_{\mathrm{MC}_{\mathrm{cor}}} .
\end{aligned}
$$

ALEPH and L3 are using a differential procedure concerning the $\mathrm{b}$ tagging used to extract the $\mathrm{b}$ charge separation in uncorrelated selected data samples of changing b purity. DELPHI and OPAL are using an integral ansatz and extract the $\mathrm{b}$ charge separation in correlated data samples. In all analyses the background contribution was estimated with simulation and subtracted to extract the $\mathrm{b}$ charge separation.

The $\delta_{\mathrm{b}}$ results of the DELPHI experiment are shown for two different values of the weighting parameter $\kappa$ and for different years of data taking [i]1]:

\begin{tabular}{c|cc} 
year & $\delta_{\mathrm{b}}(\kappa=0.5)$ & $\delta_{\mathrm{b}}(\kappa=1.0)$ \\
\hline 92 & $-0.1470 \pm 0.0029$ & $-0.2114 \pm 0.0048$ \\
93 & $-0.1463 \pm 0.0035$ & $-0.2079 \pm 0.0058$ \\
94 & $-0.1446 \pm 0.0019$ & $-0.2081 \pm 0.0032$ \\
95 & $-0.1452 \pm 0.0033$ & $-0.2069 \pm 0.0056$
\end{tabular}

Table 1: Extracted $\delta_{\mathrm{b}}$ values for different years as measured by DELPHI.

The results for the forward-backward asymmetry are then extracted by a fit to the data as a function of the polar angle using Eqn.5.2: The results of DELPHI for different years of data taking are shown in Fig. 13 i for $\kappa=0.8$ as a function of the b purity [i] is marked by the arrow and the horizontal line corresponds to the combined peak result.

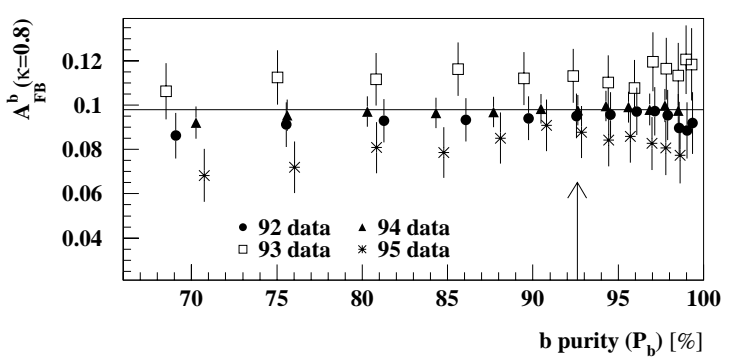

Figure 13: Results of the $b$ forward-backward asymmetry on the $\mathrm{Z}$ peak as measured by DELPHI.

As ALEPH and L3 are using the differential procedure, they can perform a fit to $A_{F B}^{\mathrm{b} \overline{\mathrm{b}}}$ not only as a function of the polar angle but also as a function of the $b$ purity. While DELPHI and OPAL are using the integral ansatz and perform only a fit in the angle. ALEPH uses in addition

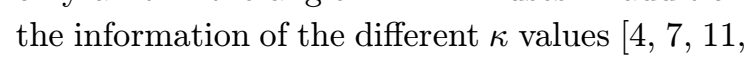
곤. 


\section{SLD - Asymmetry Measurements}

As mentioned before, the SLC collider can produce a polarized electron beam with a polarization up to $80 \%$. Therefore the SLD experiment can measure other asymmetry observables compared to the LEP experiments. While the latter always measure a mixture of the initial and the final state couplings, the former can measure the initial state and final state couplings independently.

\section{$6.1 A_{\mathrm{b}}$ measurement}

SLD performs three different analyses to measure $A_{\mathrm{b}}$ from the left-right-forward-backward asym-

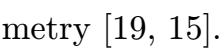

The classical method using the lepton tag and the one using the jet charge technique will not be described here. They are very similar to the methods used by the LEP experiments.

The third kind of analysis applies a kaon tag to enrich $b$ quark events originating from the $\mathrm{b} \rightarrow \mathrm{c} \rightarrow \mathrm{s}$ cascade decay. A secondary vertex can be reconstructed and the corresponding impact parameter of the kaon is used as b tagging variable. The charge of the kaon is used to distinguish between the quark and the anti-quark hemisphere. The systematic error of this analysis is dominated by the uncertainty of the branching ratio $\mathrm{BR}\left(\mathrm{B}^{-+} \rightarrow \mathrm{K}^{+-}\right)$:

The Combination of all three analyses yields: SLD result :

$$
\Rightarrow A_{\mathrm{b}}=0.866 \pm 0.036
$$

which is in good agreement with the LEP measurements,

LEP result :

$$
\Rightarrow A_{\mathrm{b}}=0.885 \pm 0.022 \text {. }
$$

The LEP number is extracted using $A_{\mathrm{e}}=0.1491 \pm$ 0.0018 as measured by SLD with the $A_{L R}$ analyses and combined with the LEP analyses of the $A_{1}$ measurement [1] $19 \bar{l}$.

\section{2 $A_{\mathrm{c}}$ measurement}

The $A_{\mathrm{c}}$ result is extracted similar to the $A_{\mathrm{b}}$ analyses by measuring the left-right-forward-backward asymmetry. Again three different approaches can be used.

The classical lepton tag analysis provides both the $A_{\mathrm{c}}$ and the $A_{\mathrm{b}}$ result. It will be not described here as it is similar to the methods used by the LEP experiments. In the second approach D mesons are reconstructed in analogy to the procedures used at LEP for the asymmetry measure-

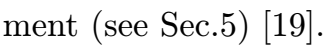

The third analysis uses an inclusive charm tag. The performance of the SLD vertex detector and $\mathrm{b}$ tagging algorithm allows to identify also $\mathrm{c}$ quark events (see Fig. (6) $_{1}^{1}$ ). The measurement of the mass and the momentum of the secondary vertex is essential for the $c$ tag variable, while only the mass of the secondary vertex is included for the $b$ tag. A charm quark event is selected requiring at least one hemisphere tagged as $\mathrm{c}$ and no hemisphere tagged as $\mathrm{b}$. The quark charge is measured in $50 \%$ of the cases by the charge of the vertex and in $25 \%$ by the charge of the kaon attached to the secondary vertex [i1 $\left.\overline{6}_{1}\right]$.

The final $A_{\mathrm{c}}$ result is achieved by combining all three analyses:

SLD result :

$$
\Rightarrow A_{\mathrm{c}}=0.649 \pm 0.040 \text {. }
$$

The LEP result is deduced in analogy to the $A_{\mathrm{b}}$ extraction by using the same $A_{\mathrm{e}}$ value [1 $\left[1 \mathrm{~g}^{\prime}\right]$.

LEP result :

$$
\Rightarrow A_{\mathrm{c}}=0.634 \pm 0.040 \text {. }
$$

Both results are in good agreement.

\section{3 $A_{L R}$ measurement}

To archive $A_{L R}$, the left- and right-handed cross sections have to be measured. In this case not only heavy quark events are used but all hadronic decays are selected. The contribution of tau events inside the selected data sample is found to be small. The $\mathrm{e}^{+} \mathrm{e}^{-}-$final state is rejected additionally because of its t-channel photon contribution [1 18,201$]$. Important for this analysis are:

- measurement of the polarization

- measurement of the luminosity and control of the beam energy

- control of the background

- measurement of the selection efficiency

The precise knowledge of the polarization, which could be increase in the last years from $63 \%$ to $80 \%$, is crucial. A Compton scattering polarimeter is used for its measurement. To reduce sys- 
tematic uncertainties, three different combinations between the electron and the photon beam parameters are used. They change with a high frequency to reduce the detector dependence. A relative error of $\delta \mathcal{P} / \mathcal{P}=0.67 \%$ could be archived for the data of 1994 and 1995, while it is slightly higher, but still under investigation, for the data taking period of 1997 and 1998: $\delta \mathcal{P} / \mathcal{P}=1.03 \%$.

The combined result using all data since 1992 is:

SLD result :

$$
\Rightarrow A_{L R}=0.1510 \pm 0.0025 \text {. }
$$

Note that the data from the $1996-1998$ period is still preliminary.

\section{Combined LEP/SLC Results}

All LEP measurements of $A_{F B}^{\mathrm{b} \overline{\mathrm{b}}}$ coming from both the classical lepton tag and the jet charge analyses are shown in Fig.14 as provided by the LEP electroweak working group [2] $\left.{ }_{1}^{1}\right]$. The variation

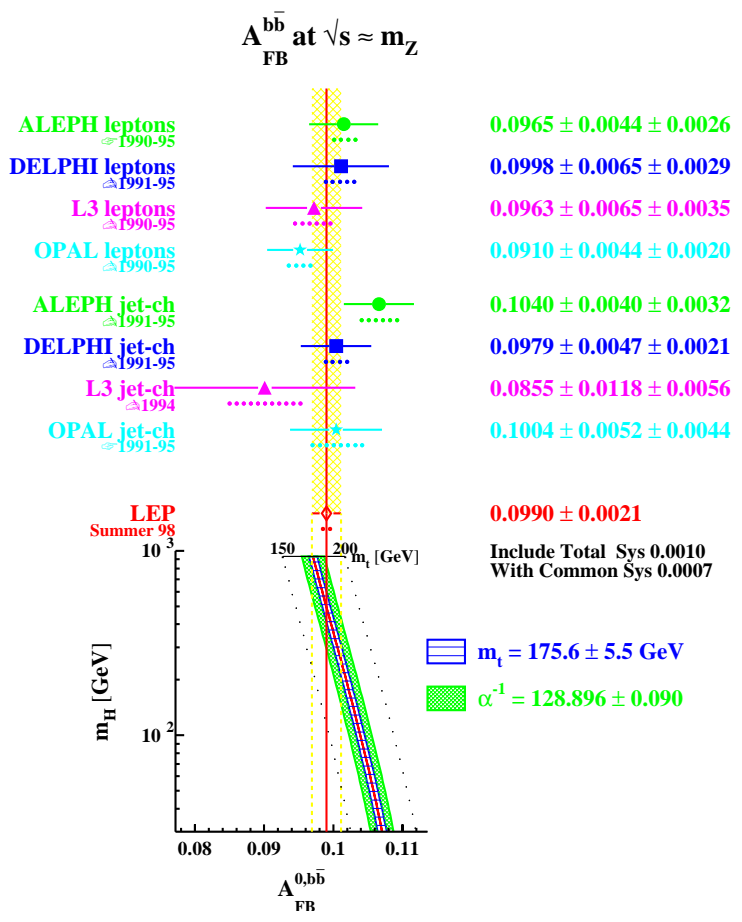

Figure 14: Summary of the LEP results for the b forward-backward asymmetry.

of the Higgs mass with the pole asymmetry for $b$ quarks is shown in the lower plot. The combined result with a final precision of $2.1 \%$ is marked by the vertical line.
A similar summary for $A_{F B}^{\mathrm{c} \bar{c}}$ is shown in Fig. $\overline{1} \overline{5}_{1}^{\prime}$, where the classical lepton measurements and the analyses with reconstructed D mesons are shown. A final precision of $6.2 \%$ for the combined LEP

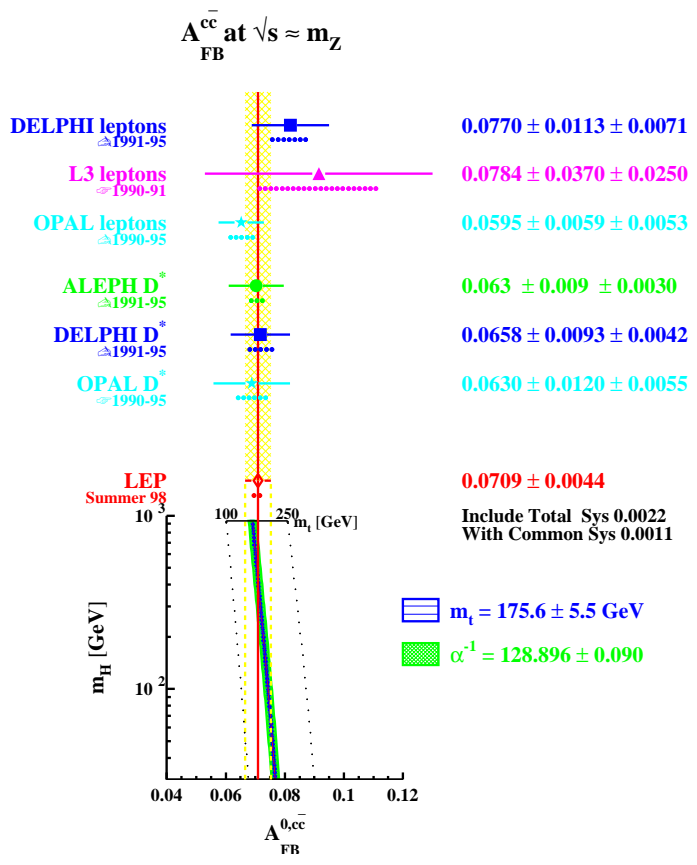

Figure 15: Summary of the LEP results for the $c$ forward-backward asymmetry.

result could be achieved.

The dependence of $A_{F B}^{\mathrm{c} \overline{\mathrm{c}}}$ and $A_{F B}^{\mathrm{b} \overline{\mathrm{b}}}$ on the centre-of-mass energy has been studied and is well described by the Standard Model prediction as can be seen in Fig. $\overline{1}_{\mathbf{1}}^{\prime \prime}$.

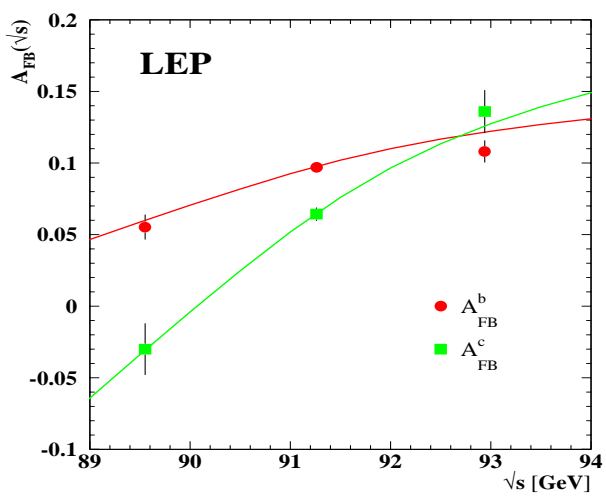

Figure 16: The energy dependence of the $c$ and b forward-backward asymmetry in comparison with the Standard Model prediction. 
Finally, the summary of all measurements contributing to $\sin ^{2} \theta_{\text {eff }}^{\text {lep }}$ is shown in Fig. $1 \bar{z}_{1}$.

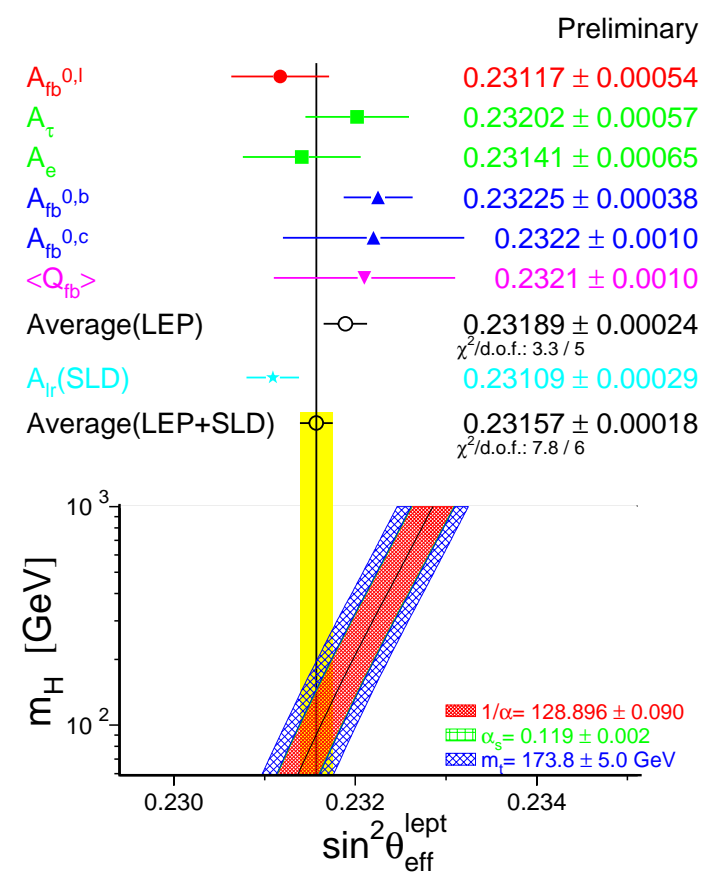

Figure 17: Summary of all electroweak mixing angle results.

The most precise results are obtained by the LEP measurement of the forward-backward asymmetry for $b$ quarks and the measurement of the left-right asymmetry as performed by SLD. Their discrepancy is of the order of $2.1 \sigma$. A high precision of $0.08 \%$ for the combined result could be achieved and a low Higgs mass is prefered by this value.

\section{Conclusion}

A review of the recent precise measurements of the weak mixing angle, $\sin ^{2} \theta_{\text {eff }}^{\text {lep }}$, by the SLD experiment at SLC and by the ALEPH, DELPHI, L3 and OPAL experiments at LEP has been given. Emphasis was put on the determination of asymmetries with heavy quarks at the $\mathrm{Z}$ pole.

The LEP and SLC results can be summarized as follows:

- The combined LEP result for $A_{F B}^{\mathrm{b} \overline{\mathrm{b}}}$ achieves a relative precision of $2.1 \%$. A difference of
$1.2 \sigma$ between the lepton and the jet charge analyses has been found.

- The combined LEP result for $A_{F B}^{c \bar{c}}$ achieves a relative precision of $6.2 \%$. This measurement is now as precise as the combined inclusive jet charge result according to the corresponding $\sin ^{2} \theta_{\text {eff }}^{\text {lep }}$ value.

- The energy dependence of $A_{F B}^{\mathrm{c} \bar{c}}$ and $A_{F B}^{\mathrm{b} \bar{b}}$ measured by LEP is in good agreement with the Standard Model expectation.

- The precision for $\sin ^{2} \theta_{\text {eff }}^{\text {lep }}$ measured at LEP is $0.10 \%$.

- The precision for $\sin ^{2} \theta_{\text {eff }}^{\text {lep }}$ measured at SLD is $0.12 \%$.

Combining and comparing both the LEP and the SLD results yields:

- Concerning the measurement of $\sin ^{2} \theta_{\mathrm{eff}}^{\mathrm{lep}}, A_{F B}^{\mathrm{b} \overline{\mathrm{b}}}$ is the most powerful LEP measurement.

- The SLD measurement of $A_{L R}$ is very competitive.

- The $A_{F B}^{c \bar{c}}$ result achieves a precision similar to the inclusive jet charge measurement, $\left\langle Q_{F B}\right\rangle$.

- A discrepancy of $2.1 \sigma$ between the SLD and LEP $\sin ^{2} \theta_{\text {eff }}^{\text {lep }}$ results remain.

\section{Acknowledgments}

It is a pleasure for me to thank all my colleagues from different experiments for providing me with their data and posting their results to the WWW. I would also like to thank A. Baroncelli and J. Drees. Both supported me and made it possible for me to come to the conference. Thanks to the organizers for their helping hands and warm hospitality. I am grateful to J. Heuser for reading the manuscript of this report and for giving useful comments to me. And finally I would like to thank G.Schwering for his patience and understanding. His suggestions and comments were a big help during the preparation of the talk and this manuscript. 
References

[18] SLD Collaboration, K. Abe et al., 'Phys. Rev:'

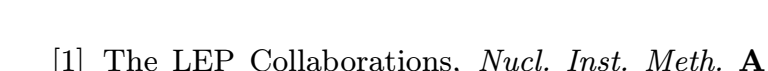
Lett. 78 (1997) 2075 378 (1996) 101

[2] K. Mönig, Status of Electroweak Tests with Heavy Quarks, Rept. Prog. Phys. 61 (1998) 999

[3] ALEPH Collaboration, D. Buskulic et al., í : - Lett. B 384 (1996) 414,

[4] ALEPH Collaboration, R. Barate et al., 'Phys.' : - Lett. B 426 (1998) 217,

[5] ALEPH Collaboration, R. Barate et al., iphys.' _ - _ Lett. B 434 (1998) 415

[6] DELPHI Collaboration, P. Abreu et al., ' $Z \bar{Z} . \mathbf{I}$ : - Physik C 65 (1995) 569 DELPHI Collaboration, P. Abreu et al., ICHEP'98\#124, paper submitted to XXIX International Conference on High Energy Physics, July 23-30,1998, Vancouver, Canada

[7] DELPHI Collaboration, P. Abreu et al., CERNEP-98-189 (1998), to be published in Eur. Phys. J. $C$

[8] DELPHI Collaboration, P. Abreu et al., hep-ex/9903074 to be published in Eur. Phys. J. $C$

_ [9] L3 Collaboration, M. Acciarri et al., 'Phys. Lett.' : - _ - 448 (1999) 152

[10] L3 Collaboration, M. Acciarri et al., 'Phys. Lètt.' B $\mathbf{3} \overline{3} \overline{5}(1994)-542$

[11] L3 Collaboration, M. Acciarri et al., 'Phys. Lett.' : _ _ - $\mathbf{4 3 9}$ (1998) 225

[12] OPAL Collaboration, G. Alexander et al., IZ.' : _ Physik C $\mathbf{7 0}(1996)$ 357

[13] OPAL Collaboration, K. Ackerstaff et al., ' $\mathbf{Z}: \mathbf{I}$ : Physik C $\mathbf{7 5}(1997)$ 385

[14] OPAL Collaboration, G. Alexander et al., ' $\bar{Z} \mathbf{I}$ Physik

[15] SLD Collaboration, K. Abe et al., SLAC-PUB7886, paper submitted to XXIX International Conference on High Energy Physics, July 2330,1998, Vancouver, Canada

[16] SLD Collaboration, K. Abe et al., SLAC-PUB7879, paper submitted to XXIX International Conference on High Energy Physics, July 2330,1998, Vancouver, Canada

[17] SLD Collaboration, K. Abe et al.,SLAC-PUB7878 , paper submitted to XXIX International Conference on High Energy Physics, July 2330,1998, Vancouver, Canada
[19] SLD Collaboration, S. Fahey, ICHEP'98, presentation at the XXIX International Conference on High Energy Physics, July 23-30,1998, Vancouver, Canada

[20] SLD Collaboration, K. Baird, ICHEP'98, presentation at the XXIX International Conference on High Energy Physics, July 23-30,1998, Vancouver, Canada

[21] The LEP Heavy Flavour Working Group, Input Parameters for LEP electroweak Heavy Flavour Results for Summer 1998 Conferences, CERNEP/99-015 (1999)

Pictures are available at: /http://www.cern.ch/LEPEWWG/plots/. 